\title{
A QUASIANALYTIC SINGULAR SPECTRUM WITH RESPECT TO THE DENJOY-CARLEMAN CLASS
}

\author{
SOON-YEONG CHUNG AND DOHAN KIM
}

\begin{abstract}
Making use of the FBI (Fourier-Bros-Iagolnitzer) transforms we simplify the quasianalytic singular spectrum for the Fourier hyperfunctions, which was defined for distributions by Hörmander as follows; for any Fourier hyperfunction $u,\left(x_{0}, \xi_{0}\right)$ does not belong to the quasianalytic singular spectrum $W F_{M}(u)$ if and only if there exist positive constants $C, \gamma$ and $N$, and a neighborhood of $x_{0}$ and a conic neighborhood $\Gamma$ of $\xi_{0}$ such that

$$
\mid u_{y}\left[\left(\exp -|\xi|(x-y)^{2} / 2-i\langle y, \xi\rangle\right] \mid \leq C \exp [-M(\gamma|\xi|)]\right.
$$

for all $x \in U,|\xi| \in \Gamma$ and $|\xi| \geq N$, where $M(t)$ is the associated function of the defining sequence $M_{p}$. This result simplifies Hörmander's definition and unify the singular spectra for the $C^{\infty}$ class, the analytic class and the DenjoyCarleman class, both quasianalytic and nonquasianalytic.
\end{abstract}

\section{$\S 0$. Introduction}

The classification of singularities according to their spectrum was developed by several mathematicians around 1970. Sato introduced and studied the analytic singular spectrum for the hyperfunctions in [Sa], and Hörmander defined $W F(u)$ for the distributions by means of pseudodifferential operators, and later by multiplying cutoff functions and taking Fourier transforms for the $C^{\infty}$ class in [H1, H4]. Hörmander also introduced the wave front set $W F_{M}(u)$ with respect to the Denjoy-Carleman class $C^{M}$ in $[\mathrm{H} 2, \mathrm{H} 4]$, which includes the analytic wave front set $W F_{A}(u)$ as a special case. To define this he used a sequence of cutoff functions to overcome the difficulty of absence of cutoff functions in the class of analytic functions. But it is rather complicated, and difficult to compare this with $C^{\infty}$ wave front set $W F(u)$. On the other hand, making use of the FBI transforms, Bros and Iagolnitzer introduced the essential spectrum, which was shown to be equal to the analytic singular spectrum of Sato and the analytic wave front set of Hörmander by Bony in [B].

Received March 26, 1996.

Partially supported by KOSEF and GARC 
The purpose of this paper is to define a singular spectrum with respect to $C^{M}$ for the Fourier hyperfunctions which include distributions and Sato's hyperfunctions in the spirit of Bros-Iagolnitzer and to unify the theories of singular spectra for the case of the analytic class, the $C^{\infty}$ class and the Denjoy-Carleman class, both quasianalytic and nonquasianalytic.

The following is the local regularity theorem for $C^{M}$ by Hörmander as in $[\mathrm{H} 2, \mathrm{H} 4]$ :

THEOREM 0.1. ([H4]) Let $u$ be a distribution. Then $u$ is $C^{M}$ near $x_{0}$ if and only if there exist a constant $C>0$, a neighborhood $U$ of $x_{0}$ and a bounded sequence $u_{p} \in \mathcal{E}^{\prime}$ which is equal to $u$ in $U$ and satisfies

$$
\left|\hat{u}_{p}(\xi)\right| \leq C\left(C^{p} M_{p} /|\xi|^{p}\right), \quad p=1,2,3, \ldots
$$

where ^ denotes the Fourier transform.

We simplify the above theorem and generalize to the larger category of Fourier hyperfunctions as a main theorem in this paper as follows:

THEOREM 0.2. Let $u$ be a Fourier hyperfunction. Then $u$ is $C^{M}$ near $x_{0}$ if and only if there are positive constants $C, \gamma, N$ and a neighborhood $U$ of $x_{0}$ such that

$$
\left|u_{y}\left(\exp \left[-|\xi|(x-y)^{2} / 2-i\langle y, \xi\rangle\right]\right)\right| \leq C \exp [-M(\gamma|\xi|)]
$$

for all $x \in U$ and $|\xi| \geq N$, where $M(t)$ is the associated function of $M_{p}$ (see (1.6) for the definition).

Also, we derive from the above theorem a local regularity theorem for the analytic class and the $C^{\infty}$ class as a corollary, which generalizes a microlocal result of Matsuzawa in $[\mathrm{M}]$.

\section{$\S 1$. Preliminaries}

We first introduce the real version of the Fourier hyperfunctions as defined in $[\mathrm{KCK}]$. We refer to $[\mathrm{Ka}]$ for the sphere compactification $\mathbb{D}^{n}$, which is $\mathbb{R}^{n} \cup S_{\infty}^{n-1}$, of $\mathbb{R}^{n}$ where $S_{\infty}^{n-1}$ is an $(n-1)$-dimensional sphere at infinity and the original definition of Sato-Kawai. 
Definition 1.1. ([KCK]) Let $K$ be a compact set in $\mathbb{D}^{n}$. We say that $\phi$ is in $\mathcal{F}(K)$ if $\phi \in C^{\infty}\left(\Omega \cap \mathbb{R}^{n}\right)$ for some neighborhood $\Omega$ of $K$ and if there are positive constants $h$ and $k$ such that

$$
|\phi|_{k, h}=\sup _{\substack{x \in \Omega \cap \mathbb{R}^{n} \\ \alpha}} \frac{\left|\partial^{\alpha} \phi(x)\right|}{h^{|\alpha|} \alpha !} \exp k|x|<\infty .
$$

Here we use the multi-index notation: $|\alpha|=\alpha_{1}+\cdots+\alpha_{n}, \partial^{\alpha}=\partial_{1}^{\alpha_{1}} \cdots \partial_{n}^{\alpha_{n}}$, $\partial_{j}=\partial / \partial x_{j}$ for $\alpha=\left(\alpha_{1}, \alpha_{2}, \cdots, \alpha_{n}\right) \in \mathbb{N}_{0}^{n}$ where $\mathbb{N}_{0}$ is the set of nonnegative integers.

We say that $\phi_{j} \rightarrow 0$ in $\mathcal{F}(K)$ as $j \rightarrow \infty$ if there are positive constants $h$ and $k$ such that

$$
\sup _{\substack{x \in \Omega \cap \mathbb{R}^{n} \\ \alpha}} \frac{\left|\partial^{\alpha} \phi_{j}(x)\right|}{h^{|\alpha|} \alpha !} \exp k|x| \rightarrow 0 \text { as } j \rightarrow \infty,
$$

where $\Omega$ is any neighborhood of $K$.

We denote by $\mathcal{F}^{\prime}(K)$ the strong dual space of $\mathcal{F}(K)$ and call its elements Fourier hyperfunctions carried by $K$. Especially if $K=\mathbb{D}^{n}$ then we often use the notation $\mathcal{F}^{\prime}$ simply instead of $\mathcal{F}^{\prime}\left(\mathbb{D}^{n}\right)$.

In fact, in $[\mathrm{KCK}]$ the space $\mathcal{F}(K)$ is shown to be topologically equivalent to the space $\mathcal{P}_{*}(\mathcal{K})$ of a holomorphic functions in a complex neighborhood $\Omega \cap \mathbb{R}^{n}+i\{|y|<r\}$ of $K$ in $\mathbb{C}^{n}$ satisfying the estimate

$$
\sup _{z \in \Omega \cap \mathbb{R}^{n}+i\{|y|<r\}}|\phi(z)| \exp k|z|<\infty
$$

for some $k$, which was originally defined by Sato-Kawai. Here $z=x+i y$ and $\Omega$ is a neighborhood of $K$ in $\mathbb{D}^{n}$.

We denote by $E(x, t)$ the $n$-dimensional heat kernel:

$$
E(x, t)= \begin{cases}(4 \pi t)^{-n / 2} \exp \left[-|x|^{2} / 4 t\right], & t>0 \\ 0, & t \leq 0\end{cases}
$$

and set $U(x, t)=u_{y}(E(x-y, t))$ for $u \in \mathcal{F}^{\prime}(K)$.

The following characterization of the Fourier hyperfunctions by the heat kernel is very useful later.

THEOREM 1.2. ([KCK]) Let $u \in \mathcal{F}^{\prime}(K)$ and let

$$
U(x, t)=u_{y}(E(x-y, t)), \quad t>0 .
$$


Then $U(x, t) \in C^{\infty}\left(\mathbb{R}_{+}^{n+1}\right), \mathbb{R}_{+}^{n+1}=\left\{(x, t) \in \mathbb{R}^{n+1} \mid x \in \mathbb{R}^{n}, t>0\right\}$ and satisfies the heat equation

$$
\left(\partial_{t}-\Delta\right) U(x, t)=0 \text { in } \mathbb{R}_{+}^{n+1} .
$$

For every $\varepsilon>0$ there exists a constant $C>0$ such that

$$
|U(x, t)| \leq C \exp \left[\varepsilon\left(\frac{1}{t}+t+|x|\right)-\frac{d\left(x, K_{\delta} \cap \mathbb{R}^{n}\right)^{2}}{8 t}\right], \quad t>0
$$

where $K_{\delta}=\{x|| x-y \mid \leq \delta$ for some $y \in K\}$.

Moreover, we have

$$
\begin{aligned}
& U(x, t) \rightarrow u \text { in } \mathcal{F}^{\prime} \text { as } t \rightarrow 0+, \text { i.e., } \\
& u(\phi)=\lim _{t \rightarrow 0+} \int U(x, t) \phi(x) d x, \quad \phi \in \mathcal{F}(K) .
\end{aligned}
$$

Conversely, every $C^{\infty}$ solution $U(x, t)$ defined in $\mathbb{R}_{+}^{n+1}$ satisfying the conditions (1.3) and (1.4) can be expressed in the form

$$
U(x, t)=u_{y}(E(x-y, t))
$$

with a unique element $u \in \mathcal{F}^{\prime}(K)$.

In particular, the heat kernel $E(x, t)$.corresponds to the Dirac measure $\delta$, since

$$
\phi(x)=\lim _{t \rightarrow 0^{+}} \int E(x-y, t) \phi(y) d y, \quad \phi \in \mathcal{F}(K) .
$$

For more details of the Fourier hyperfunctions and the related theory we refer to [KCK, Ka].

Now we would like to introduce the Denjoy-Carleman class $C^{M}$, which lies between the analytic class and the $C^{\infty}$ class.

Let $M_{p}, p=0,1,2, \ldots$, be a sequence of positive numbers and $\Omega$ be an open set in $\mathbb{R}^{n}$. We impose the following conditions on $M_{p}$ :

(M.0) There is a constant $h>0$ such that

$$
p ! \leq C h^{p} M_{p}, \quad p=0,1,2, \ldots
$$

(M.1) $M_{p}^{2} \leq M_{p-1} M_{p+1}, \quad p=1,2,3, \ldots$ 
(M.2) There are positive constants $C, H$ such that

$$
M_{p+q} \leq C H^{p+q} M_{p} M_{q}, \quad p, q=0,1,2, \ldots
$$

Definition 1.3. We denote by the Denjoy-Carleman class $C^{M}(\Omega)$ the set of all $\phi \in C^{\infty}(\Omega)$ such that on each compact set $K \subset \Omega$ its derivatives satisfy the estimates

$$
\sup _{x \in K}\left|\partial^{\alpha} \phi(x)\right| \leq C h^{|\alpha|} M_{|\alpha|}, \quad \alpha \in \mathbb{N}_{0}^{n}
$$

for some constants $C>0$ and $h>0$. Sometimes this class is called the space of ultradifferentiable functions in $\Omega$.

Actually, for the above class (M.0) means that every analytic function belongs to $C^{M}(\Omega)$ and (M.2) means that $C^{M}(\Omega)$ is stable under the ultradifferential operator. By Gorny's theorem referred in $[\mathrm{Ko}]$ we can always rearrange the sequence $M_{p}$ without any change of $C^{M}(\Omega)$ so that (M.1) should be satisfied. Therefore, the above three conditions on $M_{p}$ is the most fundamental and essential.

For each sequence $M_{p}$ given as above its associated function $M(t)$ on $[0, \infty)$ is defined as follows:

$$
M(t)=\sup _{p} \log \frac{M_{0} t^{p}}{M_{p}}
$$

and

$$
M(\xi)=M(|\xi|), \quad \xi \in \mathbb{R}^{n}
$$

Then (M.0) gives

$$
\alpha \log t \leq M(t) \leq \beta t, \quad t>0
$$

for a couple of positive constants $\alpha$ and $\beta$.

We refer to $[\mathrm{H} 4, \mathrm{Ko}]$ for more details on the Denjoy-Carleman class and the properties of the associated function.

\section{§. Main Theorem}

We first define the FBI transform $\tilde{u}(x, \xi)$ of a Fourier hyperfunction $u$ by the formula

$$
\tilde{u}(x, \xi)=u_{y}\left(\exp \left[-|\xi|(x-y)^{2} / 2-i\langle y, \xi\rangle\right]\right)
$$


for $\xi \neq 0$ and $x \in \mathbb{R}^{n}$. Since the Gaussian function is exponentially decreasing $\tilde{u}(x, \xi)$ is well defined for any Fourier hyperfunction $u$.

We are now in a position to state the local regularity theorem for $\mathcal{F}^{\prime}$ which will be used to define a singular spectrum with respect to the DenjoyCarleman class for $\mathcal{F}^{\prime}$. In fact, this is the main theorem of this paper.

Theorem 2.1. Let $u \in \mathcal{F}^{\prime}$ and $x_{0} \in \mathbb{R}^{n}$. Then $u \in C^{M}$ in a neighborhood of $x_{0}$ if and only if there are positive constants $C, \gamma, N$ and a neighborhood $U$ of $x_{0}$ such that

$$
|\tilde{u}(x, \xi)| \leq C \exp [-M(\gamma|\xi|)]
$$

for all $x \in U$ and $|\xi| \geq N$.

To prove this theorem we need the following two lemmas.

LEMMA 2.2. Let $u \in \mathcal{F}^{\prime}$ vanish near $x_{0}$. Then for any $N>0$ there exist positive constants $C, \alpha$ and a neighborhood $W$ of $x_{0}$ such that

$$
|\tilde{u}(x, \xi)| \leq C \exp [-\alpha|\xi|],
$$

for all $x \in W$ and $|\xi| \geq N$.

Thus, $\tilde{u}$ satisfies the condition (2.2).

Proof. Choose $\delta>0$ sufficiently small so that $u=0$ in a neighborhood

$$
W_{3 \delta}=\left\{x|| x-x_{0} \mid<3 \delta\right\}
$$

Since

$$
\operatorname{supp} u \subset \mathbb{D}^{n} \backslash W_{3 \delta}
$$

for any $r>0$ and $k>0$ there exists a constant $C>0$ such that

$$
|u(\phi)| \leq C \sup _{\substack{z=y+i \eta \\\left|y-x_{0}\right|>2 \delta \\|\eta| \leq r}}|\phi(z)| \exp k|z|
$$

for any $\phi \in \mathcal{F}\left(\mathbb{D}^{n}\right)$. Let $x \in W_{\delta}, r \ll \delta,|\xi| \geq N$ and $z=y+i \eta$. Then we have

$$
|\tilde{u}(x, \xi)|
$$




$$
\begin{aligned}
& \leq C \sup _{\substack{\left|y-x_{0}\right|>2 \delta \\
|\eta| \leq r}}\left|\exp \left[-|\xi|(z-x)^{2} / 2-i\langle z, \xi\rangle\right]\right| \exp k|z| \\
& \leq C \sup _{\substack{\left|y-x_{0}\right|>2 \delta \\
|\eta| \leq r}} \exp \left[-\frac{|\xi|}{2}\left\{(y-x)^{2}-\eta^{2}\right\}+\langle\eta, \xi\rangle+k(|y|+|\eta|)\right] \\
& \leq C \sup _{\left|y-x_{0}\right|>2 \delta} \exp \left[-\frac{|\xi|}{4} \delta^{2}+|\xi|\left(\frac{r^{2}}{2}+r\right)+k \delta+k\left|x_{0}\right|+k r\right. \\
& \left.\qquad-\frac{|\xi|}{4}\left\{|y-x|^{2}-\frac{2 k}{|\xi|}\right\}^{2}+\frac{k^{2}}{|\xi|}\right] \\
& \leq C \exp \left[-|\xi|\left(\frac{\delta^{2}}{4}-\frac{r^{2}+2 r}{2}\right)+k\left(\delta+\left|x_{0}\right|+r\right)+\frac{k^{2}}{N}\right] \\
& \leq C\left(k, r, x_{0}, N\right) \exp [-\alpha|\xi|]
\end{aligned}
$$

for some $\alpha=\alpha(\delta, r)$ if we choose $r>0$ so small that $\delta^{2} / 4>\left(r^{2}+2 r\right) / 2$. This completes the proof.

LEMmA 2.3. Let $u, v \in \mathcal{F}^{\prime}$ and $u=v$ in a neighborhood of $x_{0}$. Suppose $u$ satisfies (2.2). Then $v$ also satisfies (2.2).

Proof. Let $w=v-u$. Then $w$ vanishes in a neighborhood $U$ of $x_{0}$. Therefore it follows that $w$ satisfies

$$
\begin{aligned}
|\tilde{w}(x, \xi)| & \leq C_{1} \exp [-\alpha|\xi|] \\
& \leq C_{1} \exp [-M(\alpha|\xi|)]
\end{aligned}
$$

for $|\xi|>N$ and $x$ in a neighborhood $W \subset U$ of $x_{0}$. Thus we obtain

$$
\begin{aligned}
|\tilde{v}(x, \xi)| & \leq|\tilde{u}(x, \xi)|+|\tilde{w}(x, \xi)| \\
& \leq C_{2} \exp [-M(\gamma|\xi|)]+C_{1} \exp [-M(\alpha|\xi|)] \\
& \leq 2 C_{3} \exp \left[-M\left(\gamma^{\prime}|\xi|\right)\right]
\end{aligned}
$$

where $C_{3}=\max \left(C_{1}, C_{2}\right)$ and $\gamma^{\prime}=\min (\alpha, \gamma)$, which completes the proof.

We now prove the theorem.

Proof of Theorem 2.1. (i) Necessity: By the above lemmas it suffices to prove in a local context. As a result of this observation we may assume in the sequel that $u$ belongs to $C^{M}$ in a neighborhood $W_{2 \delta}$ of $x_{0}$ and vanishes on $\mathbb{R}^{n} \backslash W_{\delta}$. 
Then using integration by parts we have

$$
\begin{aligned}
& \xi^{\alpha} \tilde{u}(x, \xi) \\
= & \int_{W_{\delta}} u(y) \exp \left[-|\xi|(x-y)^{2} / 2\right] \xi^{\alpha} \exp (-i\langle y, \xi\rangle) d y \\
= & \int_{W_{\delta}} u(y) \exp \left[-|\xi|(x-y)^{2} / 2\right] i^{|\alpha|} \partial_{y}^{\alpha} \exp (-i\langle y, \xi\rangle) d y \\
= & \int_{W_{\delta}}(-1)^{|\alpha|} \partial^{\alpha}\left\{u(y) \exp \left[-|\xi|(x-y)^{2} / 2\right]\right\} i^{|\alpha|} \exp (-i\langle y, \xi\rangle) d y .
\end{aligned}
$$

Making use of the fact

$$
\sup _{x}\left|\partial_{x}^{\alpha} \exp \left[-s x^{2}\right]\right| \leq C_{1}^{|\alpha|} s^{|\alpha| / 2} \alpha !^{1 / 2}, \quad s>0
$$

we obtain that for $|\xi| \geq 1$

$$
\begin{aligned}
& \left|\partial_{y}^{\alpha}\left\{u(y) \exp \left[-|\xi|(x-y)^{2} / 2\right]\right\}\right| \\
\leq & \sum_{\beta \leq \alpha}\left(\begin{array}{c}
\alpha \\
\beta
\end{array}\right)\left|\partial^{\alpha-\beta} u(x)\right| \mid \partial^{\beta} \exp \left[-|\xi|(x-y)^{2} / 2\right] \\
\leq & \sum_{\beta \leq \alpha}\left(\begin{array}{c}
\alpha \\
\beta
\end{array}\right) C h^{|\alpha-\beta|} M_{|\alpha-\beta|} C_{1}^{|\beta|}(|\xi| / 2)^{|\beta| / 2} \beta !^{1 / 2} \\
\leq & C_{2}^{|\alpha|} \sum_{\beta \leq \alpha}\left(\begin{array}{c}
\alpha \\
\beta
\end{array}\right) M_{|\alpha|-|\beta|}|\xi|^{|\beta| / 2} \beta !^{1 / 2} \\
\leq & C_{3}^{|\alpha|} M_{|\alpha|} \exp (M(|\xi|) / 2),
\end{aligned}
$$

where the last inequality follows from (M.1).

Then it follows that for $x \in W_{\delta}$

$$
\begin{aligned}
|\tilde{u}(x, \xi)| & \leq C \inf _{\alpha} \frac{C_{3}^{|\alpha|} M_{|\alpha|}}{\left|\xi^{\alpha}\right|} \exp [M(|\xi|) / 2] \\
& \leq C \inf _{p} \frac{M_{p}}{(A|\xi|)^{p}} \exp [M(|\xi|) / 2] \\
& \leq C^{\prime} \exp [-M(A|\xi|)+M(|\xi| / 2)] .
\end{aligned}
$$

Making use of the inequality

$$
\frac{3}{2} M(A|\xi|)-M(|\xi|) \geq 0, \quad|\xi| \geq 1 / A^{3}
$$


we have, for $|\xi| \geq \max \left(1,1 / A^{3}\right)$

$$
|\tilde{u}(x, \xi)| \leq C \exp [-M(A|\xi|) / 4] .
$$

Therefore, if $M_{p}$ satisfies (M.2) then we have

$$
|\tilde{u}(x, \xi)| \leq C^{\prime} \exp [-M(\gamma|\xi|)]
$$

(ii) Sufficiency: Since we need only the local regularity of $u$ near $x_{0}$ we may assume that $u=0$ outside of a neighborhood $U$ of $x_{0}$. Then by Lemma 2.3 the condition (2.2) remains still valid.

We follow the context given in the proof of Lemma 2.2 in $[\mathrm{S}]$.

Since

$$
(|\xi| / 2 \pi)^{n / 2} \int_{\mathbb{R}^{n}} \exp \left[-|\xi|(s-y)^{2} / 2\right] d s=1
$$

and for $t>0$

$$
(2 \pi)^{-n} \int_{\mathbb{R}^{n}} \exp \left[-t|\xi|^{2}-i\langle x, \xi\rangle\right] d \xi=(4 \pi t)^{-n / 2} \exp \left(-|x|^{2} / 4 t\right)
$$

we can express the heat kernel $E(x-y, t)$ by the integral

(2.3) $E(x-y, t)$

$$
=(2 \pi)^{-n} \iint(|\xi| / 2 \pi)^{n / 2} \exp \left[-|\xi|(s-y)^{2} / 2-i\langle x-y, \xi\rangle\right] e^{-t|\xi|^{2}} d s d \xi .
$$

Then it follows from Theorem 1.2 that

$$
\begin{aligned}
& \delta(x-y) \\
= & (2 \pi)^{-n} \iint(|\xi| / 2 \pi)^{n / 2} \exp \left[-|\xi|(s-y)^{2} / 2-i\langle x-y, \xi\rangle\right] d s d \xi
\end{aligned}
$$

in the oscillatory sense for the Fourier hyperfunctions. In other words, for every $\phi \in \mathcal{F}$ we have

$$
\begin{aligned}
& \lim _{t \rightarrow 0^{+}} \int E(x-y, t) \phi(y) d y \\
= & \lim _{t \rightarrow 0^{+}}(2 \pi)^{-n} \iiint(|\xi| / 2 \pi)^{n / 2} \exp \left[-|\xi|(s-y)^{2} / 2-i\langle x-y, \xi\rangle\right] \\
& \quad \cdot \exp \left[-t|\xi|^{2}\right] \phi(y) d s d \xi d y \\
= & (\delta(x-y), \phi(y))
\end{aligned}
$$


Moreover, for any $N>0$ we obtain from (2.4) that

$$
\begin{aligned}
& \delta(x-y) \\
= & (2 \pi)^{-n} \iint_{|\xi| \geq N}(|\xi| / 2 \pi)^{n / 2} \exp \left[-|\xi|(s-y)^{2} / 2-i\langle x-y, \xi\rangle\right] d s d \xi \\
& +g(x, y)
\end{aligned}
$$

with some entire function $g$. Then it follows that the second term in (2.6) is analytic for $x \neq y$. In particular, consider

$$
\begin{aligned}
& h(x, y) \\
= & (2 \pi)^{-n} \iint_{\substack{\left|s-x_{0}\right| \geq 2 \varepsilon \\
|\xi| \geq N}}(|\xi| / 2 \pi)^{n / 2} \exp \left[-|\xi|(s-y)^{2} / 2-i\langle x-y, \xi\rangle\right] d s d \xi .
\end{aligned}
$$

Then by the same reason in the proof of Lemma 2.2 in [S], $h(x, y)$ is analytic in $\left\{(x, y) \in \mathbb{R}^{2 n}|| x-x_{0} \mid<\varepsilon\right\}$. Thus we have, in $\left|x-x_{0}\right|<\varepsilon$,

(2.7) $\delta(x-y)$

$$
\begin{aligned}
= & (2 \pi)^{-n} \iint_{\substack{\left|s-x_{0}\right| \leq 2 \varepsilon \\
|\xi| \geq N}}(|\xi| / 2 \pi)^{n / 2} \exp \left[-|\xi|(s-y)^{2} / 2-i\langle x-y, \xi\rangle\right] d s d \xi \\
& +k_{\varepsilon}(x, y)
\end{aligned}
$$

with some analytic function $k_{\varepsilon}(x, y)$ in the region $\left\{(x, y) \in \mathbb{R}^{2 n}|| x-x_{0} \mid<\right.$ $\varepsilon\}$.

Since $u$ vanishes outside of $U$ we can regard $u$ as an analytic functional with carrier in $U$. Thus

$$
\begin{aligned}
& u(x)=(2 \pi)^{-n} \iint_{\substack{\left|s-x_{0}\right| \leq 2 \varepsilon \\
|\xi| \geq N}}(|\xi| / 2 \pi)^{n / 2} u_{y}\left(\operatorname { e x p } \left[-|\xi|(s-y)^{2} / 2\right.\right. \\
&-i\langle x-y, \xi\rangle]) d s d \xi+w_{\varepsilon}(x) \\
&=(2 \pi)^{-n} \iint_{\substack{\left|s-x_{0}\right| \leq 2 \varepsilon \\
|\xi| \geq N}}(|\xi| / 2 \pi)^{n / 2} \tilde{u}(s, \xi) \exp [-i\langle x, \xi\rangle] d s d \xi \\
&+w_{\varepsilon}(x)
\end{aligned}
$$

where $w_{\varepsilon}(x)$ is an analytic function in $\left|x-x_{0}\right|<\varepsilon$ and $\varepsilon>0$ is a sufficiently small number.

On the other hand, since we have under the condition (M.1)

$$
\sup _{\xi} \exp [-M(\gamma|\xi|)]|\xi|^{|\alpha|}=(1 / \gamma)^{|\alpha|} M_{|\alpha|}
$$


it follows from $(2.2),(2.8)$ and $(\mathrm{M} .2)^{\prime}$ that

$$
\begin{aligned}
& \left|\frac{\partial^{\alpha}}{\partial x^{\alpha}} \iint_{\substack{\left|s-x_{0}\right| \leq 2 \varepsilon \\
|\xi| \geq N}}(|\xi| / 2 \pi)^{n / 2} \tilde{u}(s, \xi) \exp [-i\langle x, \xi\rangle] d s d \xi\right| \\
& \quad \leq C \iint_{\substack{\left|s-x_{0}\right| \leq 2 \varepsilon \\
|\xi| \geq N}}(|\xi| / 2 \pi)^{n / 2} \exp [-M(\gamma|\xi|)]|\xi|^{|\alpha|} d s d \xi \\
& \quad \leq C^{\prime}(1 / \gamma)^{|\alpha|+\frac{n}{2}} M_{|\alpha|+\frac{n}{2}} \\
& \quad \leq C\left(h^{\prime} / \gamma\right)^{|\alpha|} M_{|\alpha|}
\end{aligned}
$$

for some constants $C=C(n)>0$ and $h^{\prime}>0$. Therefore, there exist constants $C>0$ and $h>0$

$$
\left|\partial^{\alpha} u(x)\right| \leq C h^{|\alpha|} M_{|\alpha|}, \quad \alpha \in \mathbb{N}_{0}^{n}
$$

for all $x$ in the region $\left|x-x_{0}\right|<\varepsilon$, which means that $u$ belongs to $C^{M}$ in $\left|x-x_{0}\right|<\varepsilon$. This completes the proof.

Corollary 2.4. (i) Let $u \in \mathcal{F}^{\prime}$ and $x_{0} \in \mathbb{R}^{n}$. Then $u$ is real analytic in a neighborhood of $x_{0}$ if and only if there are positive constants $C, \gamma, N$ and a neighborhood $U$ of $x_{0}$ such that

$$
|\tilde{u}(x, \xi)| \leq C \exp [-\gamma|\xi|]
$$

for all $x \in U$ and $|\xi| \geq N$.

(ii) Let $u \in \mathcal{F}^{\prime}$ and $x_{0} \in \mathbb{R}^{n}$. Then $u$ is infinitely differentiable in a neighborhood of $x_{0}$ if and only if for any $k$ there exist positive constants $C_{k}, N$ and a neighborhood $U$ of $x_{0}$ such that

$$
|\tilde{u}(x, \xi)| \leq C_{k}|\xi|^{-k}, \quad k=1,2, \ldots
$$

for all $x \in U$ and $|\xi| \geq N$.

Proof. (i) Since $M_{p}=p$ ! in this case every function $\phi \in C^{M}$ is real analytic and $M(t)=t$. Thus the condition (2.2) just becomes (2.9).

(ii) Since $M_{p}=\infty$ for all $p$ except a finite number of $p$ every function $\phi \in C^{M}$ is simply infinitely differentiable and the condition (2.2) becomes (2.10), which completes the proof. 
Remark. In view of the above corollary Theorem 2.1 does not exclude the case of analytic functions or that of $C^{\infty}$ functions. Thus the advantage of our approach is to unify the theory of local regularity with respect to differentiability, analyticity and the Denjoy-Carleman class, both quasianalytic and non-quasianalytic.

We can now introduce a singular spectrum with respect to the DenjoyCarleman class $W F_{M}(u)$ for the Fourier hyperfunction $u$ as follows:

Definition 2.5. Let $u \in \mathcal{F}^{\prime}$. Then we denote by $W F_{M}(u)$ the complement of the set of $\left(x_{0}, \xi_{0}\right)$ such that there exist a neighborhood $U$ of $x_{0}$ and a conic neighborhood $\Gamma$ of $\xi_{0}$ such that for some positive constants $C, \gamma$ and $N$,

$$
|\tilde{u}(x, \xi)| \leq C \exp [-M(\gamma|\xi|)]
$$

for all $x \in U$ and $\xi \in \Gamma \cap\left\{\xi \in \mathbb{R}^{n}|| \xi \mid \geq N\right\}$.

In general, if $\sum_{p=1}^{\infty} M_{p-1} / M_{p}=\infty$, that is, it is quasianalytic then the space $C^{M}$ has no cutoff functions. In [H4], to avoid this difficulty Hörmander chose some sequence of cutoff functions with adequate bounds for derivatives up to a certain order only, which leads to a more complicated description than our results.

By the above definition of $W F_{M}(u)$ and Theorem 2.1 we easily obtain the following:

Corollary 2.6. Let $u \in \mathcal{F}^{\prime}$. Then the projection of $W F_{M}(u)$ in the space variables is equal to $\operatorname{sing} \operatorname{supp}_{M} u$.

\section{REFERENCES}

[B] J. M. Bony, Equivalence des diverses notions de spectre singulier analytique, Sém. Goulaouic-Schwartz 1975-1976, Exposé no. 3.

[CCK] J. Chung, S.-Y. Chung and D. Kim, A characterization for Fourier hyperfunctions, Publ. RIMS, Kyoto Univ., 30 (1994), 203-208.

[H1] L. Hörmander, Fourier integral operators I, Acta. Math., 127 (1971), 79-183.

[H2] _ _ Uniqueness theorems and wave front sets for solutions of linear partial differential equations with analytic coefficients, Comm. Pure Appl. Math., 24 (1971), 671-704.

[H3] — Between distributions and hyperfunctions, Astérisque, 131 (1985), 89106. 
[H4] The analysis of linear partial differential operators I, Springer-Verlag, Berlin-New York, 1983.

[I] D. Iagolnitzer, Microlocal essential support of distribution and decomposition theorem -an introduction, Lecture Notes in Math., vol. 449, Springer-Verlag, Berlin and New York, 1975, 121-132.

[Ka] T. Kawai, On the theory of Fourier hyperfunctions and its applications to partial differential equations with constant coefficients, J. Fac. Sci. Univ. Tokyo, Sect. IA, 17 (1970), 467-517.

[KCK] K.W. Kim, S.Y. Chung and D. Kim, Fourier hyperfunctions as the boundary values of smooth solutions of the heat equation, Publ. RIMS, Kyoto Univ., 29 (1993), 289-300.

[Ko] H. Komatsu, Ultradistributions I, J. Fac. Sci. Univ. Tokyo, Sect IA, 20 (1973), 25-105.

[KP] S. Krantz and H. Parks, A primer of real analytic functions, Birkhäuser, Basel, 1992.

[M] T. Matsuzawa, II, Trans. Amer. Math. Soc., 313 (1989), 619-654.

[Sa] M. Sato, Hyperfunctions and partial differential equations, Proc. Int. Conf. on Funct. Anal. and Rel. Topics, Univ. of Tokyo Press, Tokyo, 1969, pp. 91-94.

[S] J. Sjöstrand, Propagation of analytic singularities for second order Dirichlet problems, Comm. Partial Differential Equations, 5 (1980), 41-94.

Soon-Yeong Chung

Department of Mathematics

Sogang University

Seoul 121-742

Korea

sychung@ccs.sogang.ac.kr

Dohan Kim

Department of Mathematics

Seoul National University

Seoul 151-742

Korea

dhkim@math.snu.ac.kr 